\title{
Metabolite Triplet in Serum Improves the Diagnostic Accuracy of \\ Prediabetes and Diabetes Screening
}

Lichao Wang ${ }^{1,2,3 \#}$, Yinan Zhang ${ }^{4 \#}$, Xinyu $\mathrm{Liu}^{2}$, Xinjie Zhao ${ }^{2}$, Yang Ouyang ${ }^{2,3}$, Gaokun Qius, Wangjie Lv²,3, Fujian Zheng',3, QingQing Wang2,3, Xin Lu², Xiaojun Peng ${ }^{1}$, Tangchun $\mathrm{Wu}^{5}$, Rainer Lehmann ${ }^{6,7,8}$, Congrong Wang ${ }^{4,9 *}$, Weiping $\mathrm{Jia}^{4 *}$, Guowang $\mathrm{Xu}^{2 *}$

${ }^{1}$ State Key Laboratory of Fine Chemicals, Dalian University of Technology, Dalian 116023, China.

${ }^{2}$ CAS Key Laboratory of Separation Science for Analytical Chemistry, Dalian Institute of Chemical Physics, Chinese Academy of Sciences, 457 Zhongshan Road, Dalian 116023, China.

${ }^{3}$ University of Chinese Academy of Sciences, Beijing 100049, China.

${ }^{4}$ Shanghai Diabetes Institute, Shanghai Key Laboratory of Diabetes Mellitus, Shanghai Clinical Center for Endocrine and Metabolic Diseases, Metabolic diseases biobank, Shanghai Jiao Tong University Affiliated Sixth People's Hospital, Shanghai 200233, China.

${ }^{5}$ MOE Key Lab of Environment and Health, School of Public Health, Tongii Medical College, Huazhong University of Science \& Technology, Wuhan 430030, Hubei, China.

${ }^{6}$ Institute for Clinical Chemistry and Pathobiochemistry, University Hospital Tuebingen, Hoppe-Seyler-Strasse 3, 72076 Tuebingen, Germany

${ }^{7}$ Institute for Diabetes Research and Metabolic Diseases of the Helmholtz Zentrum Muenchen at the University of Tuebingen, Tuebingen, Germany

${ }^{8}$ German Center for Diabetes Research (DZD), Tübingen, Germany 
9 Shanghai East Hospital, Tongji University School of Medicine, Department of Endocrinology and Metabolic Disease, Translational Medical Center for Stem Cell Therapy, Shanghai 200120, China.

*: Corresponding authors and persons to whom reprint requests should be addressed:

Prof. Dr. Guowang Xu, E-mail: xugw@dicp.ac.cn.

Prof. Congrong Wang, E-mail: crwang@tongji.edu.cn .

Prof. Weiping Jia, E-mail: wpjia@sjtu.edu.cn .

\#: equal contribution. 


\section{List of Supporting Information}

\section{Methods}

\section{Results}

Figure S1--Discriminant analysis of discovery set 1. A) Partial least squares discriminant analysis (PLS-DA) scores plot based on normal glucose tolerant individuals, prediabetes (PreD), and diabetes. B) Validation of the PLS-DA model.

Figure S2--ROC curves of FPG, Met-T and a combination of Met-T+FPG to discriminate $\mathrm{A}$ and $\mathrm{B}$ ) normal glucose tolerant individuals from prediabetes and diabetes in discovery set 2 and validation set 1 , respectively.

Figure S3--Analytical accuracy of the LC-MS/MS method developed for the profiling of 108 diabetes related metabolites. Shown are the distributions of the relative standard deviations (RSD). A) Intra-day precision, B) Inter-day precision.

Table S1--Details of the references used to extract 108 (pre)diabetes related metabolites for knowledge-based metabolite profiling.

Table S2--List and mass spectrometric details of 108 knowledge-based selected metabolites investigated by the targeted LC-MS method in positive and negative ionization modes.

Table S3--Detailed information about IS in methanol

Table S4--Detailed information about the method validation 


\section{Methods}

\section{Chemicals and reagents}

Chromatography-grade acetonitrile and methanol were purchased from Merck (Merck, Darmstadt, Germany); Milli-Q system (Millipore, Billerica, MA, U.S.A) was used to prepare ultrapure water; ammonium acetate and acetic acid were obtained from Sigma Aldrich (St. Louis, MO, U.S.A). Internal standards (IS) including phenylalanine-d5, proline-d7, glutamine-d5, cholic acid-d4, succinic acid- ${ }^{13} \mathrm{C} 4$, hippuric acid-d5, acetyl-carnitine-d3, decanoyl-carnitine-d3, choline-d4 were also purchased from Sigma Aldrich. They were dissolved in methanol. For details about IS concentrations etc. see Table S3.

\section{HPLC-MS analysis of $\mathbf{1 0 8}$ metabolites}

\subsection{Serum sample preparation}

The serum samples were stored at $-80^{\circ} \mathrm{C}$ until analysis, thawed on ice, $200 \mu \mathrm{L}$ of ice-cold methanol containing ISs mentioned above was added to $50 \mu \mathrm{L}$ of serum in $1.5 \mathrm{~mL}$ Eppendorf tube. After vortexed for $0.5 \mathrm{~min}$, the mixture was placed on ice for $10 \mathrm{~min}$. Then the mixture was centrifuged at $4{ }^{\circ} \mathrm{C}$ for $15 \mathrm{~min}$ with a speed of $14,000 \mathrm{~g}$. $200 \mu \mathrm{L}$ of supernatant was transferred to a new Eppendorf tube and then lyophilized in CentriVap Centrifugal Vacuum Concentrators (Labconco, MO, U.S.A) and stored at $-80^{\circ} \mathrm{C}$ until analysis. Fifty $\mu \mathrm{L}$ of acetonitrile/water $1: 1$ (vol./vol.) was add to the lyophilized sample. After vortexed for $0.5 \mathrm{~min}$ and centrifuged at $4{ }^{\circ} \mathrm{C}$ for $10 \mathrm{~min}$ with a speed of $14,000 \mathrm{~g}$, the supernatant was transferred to glass vial for LC-MS analysis.

Pooled quality control samples (QCs) were acquired by mixing equivalent volume of sera from normal glucose tolerant individuals, prediabetes and diabetes patients. Pooled Lab QC was used to prepare instrument quality control samples 
(IQCs). All steps of QC metabolite extraction were the same as described above.

\subsection{Data acquisition by UHPLC-Q-trap MS system}

Serum metabolite detection was accomplished by ultra-high-performance liquid chromatography (AB SCIEX, Framingham, MA, U.S.A) hyphenated with AB SCIEX Q-trap 6500 mass spectrometer system (AB SCIEX, Framingham, MA, U.S.A). Metabolites were separated on a ZIC c-HILIC column $(2.1 \mathrm{~mm} \times 50 \mathrm{~mm} \times 3.5 \mu \mathrm{m}$, Merck) in both positive (ESI+) and negative (ESI-) modes. Column temperature was maintained at $40{ }^{\circ} \mathrm{C}$, and the flow rate was set at $0.3 \mathrm{~mL} / \mathrm{min}$. Acetonitrile/water 19:1 (vol./vol.) was set as mobile phase A containing $10 \mathrm{mM}$ ammonium acetate and $0.02 \%$ (vol./vol.) acetic acid. Mobile phase B was pure water with $10 \mathrm{mM}$ ammonium acetate and $0.02 \%$ (vol./vol.) acetic acid. The gradient was started from $5 \% \mathrm{~B}$, and linearly increased to $55 \% \mathrm{~B}$ in $6 \mathrm{~min}$, then kept for $1 \mathrm{~min}$, returned to $5 \% \mathrm{~B}$ in $0.1 \mathrm{~min}$ and equilibrated at 5\% $\mathrm{B}$ in next 3.9 min. During batch analysis, blank, IQC, QC samples were analyzed after every 10 samples. Samples in each group were analyzed with a random order. Injection volume was $1 \mu \mathrm{L}$ for all samples.

Dynamic scheduled Multiple Reaction Monitoring (MRM) mode was performed on Q-trap system for metabolite detection. The ion spray source parameters settings in ESI+ or ESI- mode were: temperature of electrospray ion source, $500{ }^{\circ} \mathrm{C}$; curtain gas, $40 \mathrm{psi}$; ion source gas1, $50 \mathrm{psi}$; ion source gas $2,50 \mathrm{psi}$; ionspary voltage, $5500 \mathrm{~V}(+)$ and $-4500 \mathrm{~V}(-)$; target scan time, 0.8s; MRM detection window, 60s; duration time, $7.6 \mathrm{~min}$.

\subsection{HPLC-MS/MS method development and validation}

In order to find reliable and practical biomarkers for rapid diabetes screening, 108 
metabolites which are closely related to diabetes were chosen for this study, from the literature including previous own publications (for a detailed list of references etc. see Table S1). A targeted method was developed to detect those target metabolites, including amino acids, carnitines, organic acids, carbohydrates, bile acids, amides and others (Table S2). Considering the polarities of these metabolites, hydrophilic interaction liquid chromatography (HILIC) was chosen. A short chromatographic column was selected allowing fast gradients and short analysis time, thereby opening perspectives to shorten total analysis time of the discovered diagnostic pattern further, i.e. to increase sample throughput for future large-scale (pre)diabetes screening studies. Systematic method validation was performed for the established method, including linearity, recovery, precision, limit of detection (LOD), and limit of quantitation (LOQ) by using pooled serum samples from healthy individuals.

Linearity was evaluated by spiking IS with different volumes into serum matrix to construct a series of 14 concentration levels. Three replicates were carried out for each point. LOD and LOQ were defined by signal/noise rate $(\mathrm{s} / \mathrm{n})=3$ and 10 , respectively. The regression coefficients (R2) of IS were $>0.99$. The results are presented in Table S4.

Recovery was calculated by the signal response rate of IS added into serum before and after extraction at each concentration (low, median, high). Each level was studied by five replicates. The recovery rate ranged from $88.1 \%$ to $111.8 \%$ and the RSD of recovery at each level was less than 10\%. Details are shown in Table S4.

Precision was evaluated by intraday and interday precision. Six replicates were performed in one day for intraday precision and three consecutive days for interday precision. Responses of all the metabolites were normalized by IS and the coefficient of variation was calculated. More than $95 \%$ metabolites had a $\mathrm{RSD} \%<20 \%$ in 
intraday precision and about $85 \%$ metabolites had a $\mathrm{RSD} \%<20 \%$ in interday precision (Figure S3).

To further study the differential metabolites, we also applied a targeted LC-MS method for 34 metabolites. Each metabolite had two MRM transitions for analysis. One was used for aiding qualitative detection and another was for quantitative detection.

\section{Nontargeted LC-MS analysis of 1,000 serum samples from nested cohort}

\subsection{Serum sample preparation and data acquisition}

One thousand serum samples were collected for prospective study from the Dongfeng Tongji cohort [S1], they were analyzed in an untargeted LC-MS method. Fifty $\mu \mathrm{L}$ serum added with 4-fold volume of methanol were mixed in 96-well plates. After vortexing for $10 \mathrm{~min}$, the extracts passed through the plates by centrifugation and then lyophilized in CentriVap Centrifugal Vacuum Concentrators. Fifty $\mu \mathrm{L}$ of methanol/water 1:4 (vol./vol.) was add to the dried residue to redissolve the metabolites and data acquisition was accomplished by ultra-high-performance liquid chromatography (UHPLC) (Waters Corp, Milford, MA, USA) hyphenated with Q Exactive HF mass spectrometer (Thermo Fisher Scientific, Bremen, Germany). Details are given in a previous paper [S2].

\subsection{Data processing}

Peak detection and alignment of metabolites were performed by TraceFinder software (Thermo Fisher Scientific, Bremen, Germany) through retention time and accurate $\mathrm{m} / \mathrm{z}$ (hexose: 179.0561; 2-hydroxybutyric acid/2-hydroxyisobutyric acid: 103.0401; phenylalanine: 164.0717). The peak areas of hexose, 2-hydroxybutyric 
acid/2-hydroxyisobutyric acid, and phenylalanine were normalized by tryptophan-d5, cholic acid-d4, and phenylalanine- $\mathrm{d} 5$ to acquire their relative concentrations, then they were used for further statistical analysis.

\section{Measurement of clinical routine laboratory parameters}

Plasma glucose level was assessed using a glucose-oxidase method (HITACHI 7600, Japan), serum insulin level was assessed using a chemical luminescence method (Roche Cobas e 601, Switzerland), HbA1c level was measured using HPLC method (Bio-rad Variant II Turbo, California, USA). For OGTT-derived indices of insulin secretion, the homeostasis model assessment of beta-cell function (HOMA-B) were calculated as $20 * \mathrm{FIN}(\mathrm{mU} / \mathrm{L}) /[\mathrm{FPG}(\mathrm{mmol} / \mathrm{L})-3.5]$. For OGTT-derived indices of insulin sensitivity, the homeostasis model assessment of insulin resistance (HOMA-IR) were calculated as FIN(mU/L)*FPG(mmol/L)/22.5.

\section{Oral glucose tolerance test}

A standard $75 \mathrm{~g}$ OGTT was administered after an 8 hour overnight fasting and venous blood samples were obtained at $0,30,60,120$ and 180 min after the start of the OGTT. The diabetes patients were diagnosed as follows: fasting plasma glucose $\geqslant 7.0 \mathrm{mmol} / \mathrm{L}$ or $2 \mathrm{~h}$ plasma glucose $\geqslant 11.1 \mathrm{mmol} / \mathrm{L}$ during a standard OGTT. The prediabetes diagnosis criteria were as follows: subjects with fasting plasma glucose $<$ $7.0 \mathrm{mmol} / \mathrm{L}$ and $7.8 \mathrm{mmol} / \mathrm{L} \leqslant 2 \mathrm{~h}$ OGTT plasma glucose $<11.1 \mathrm{mmol} / \mathrm{L}$ were defined as impaired glucose tolerance (IGT); subjects with $6.1 \mathrm{mmol} / \mathrm{L} \leqslant$ fasting glucose $<7 \mathrm{mmol} / \mathrm{L}$ and $2 \mathrm{~h}$ OGTT plasma glucose $<7.8 \mathrm{mmol} / \mathrm{L}$ were defined as impaired fasting glucose (IFG). Subjects with normal glucose tolerance had a fasting glucose $<6.1 \mathrm{mmol} / \mathrm{L}$ and $2 \mathrm{~h}$ OGTT plasma glucose $<7.8 \mathrm{mmol} / \mathrm{L}$. 


\section{Results}

\section{The models for diabetes and prediabetes screening}

The discrimination of metabolic healthy controls from prediabetes and diabetes was completed by the binary logistic regression. In model 1 , Met-T was used to construct equation 1 :

$\operatorname{logit}[\mathrm{P}($ prediabetes and diabetes $) /(1-\mathrm{P}($ prediabetes and diabetes $))]=0.2676 \times$ [phenylalanine $]+0.06146 \times[2$-hydroxybutyric/2-hydroxybutyric acid $]+0.008884$

$\times[$ hexose $]-15.5644$

In model 2, Met-T combined with FPG was used to construct equation 2:

$\operatorname{logit}[\mathrm{P}($ prediabetes and diabetes $) /(1-\mathrm{P}($ prediabetes and diabetes $))]=0.1589 \times$ [phenylalanine $]+0.08689 \times[2$-hydroxybutyric/2-hydroxybutyric acid $]+0.003651 \times$ $[$ hexose $]+2.0419 \times[\mathrm{FPG}]-19.9639$, where the $[\mathrm{P}($ prediabetes and diabetes $)]$ means the predictive probability of prediabetes and diabetes; [phenylalanine], [2-hydroxybutyric/2-hydroxybutyric acid], [hexose] and [FPG] mean the detected serum concentrations of phenylalanine, 2-hydroxybutyric/2-hydroxybutyric acid, hexose by LC-MS/MS and FPG by a glucose-oxidase method. The cut-off value of [P(prediabetes and diabetes)] in model 1 and model 2 were 0.7607 and 0.7574 , respectively.

\section{References}

[S1]. Wang F, Zhu J, Yao P, et al.; Cohort Profile: the Dongfeng-Tongji cohort study of retired workers. Int J Epidemiol 2013;42:731-740

[S2]. Ouyang Y, Tong H, Luo P, et al.; A high throughput metabolomics method and its application in female serum samples in a normal menstrual cycle based on liquid 
chromatography-mass spectrometry. Talanta 2018;185:483-490
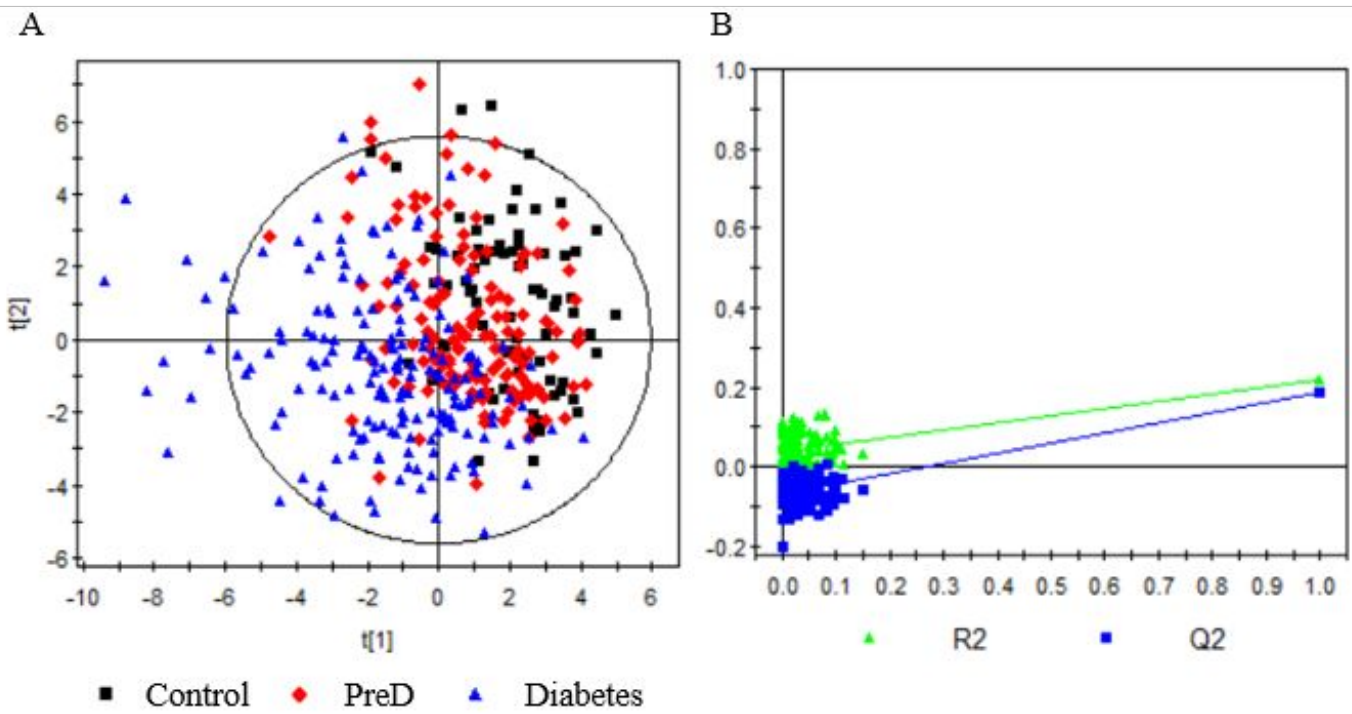

Figure S1--Discriminant analysis of discovery set 1. A) Partial least squares discriminant analysis (PLS-DA) scores plot based on normal glucose tolerant individuals, prediabetes (PreD), and diabetes. B) Validation of the PLS-DA model. 
A

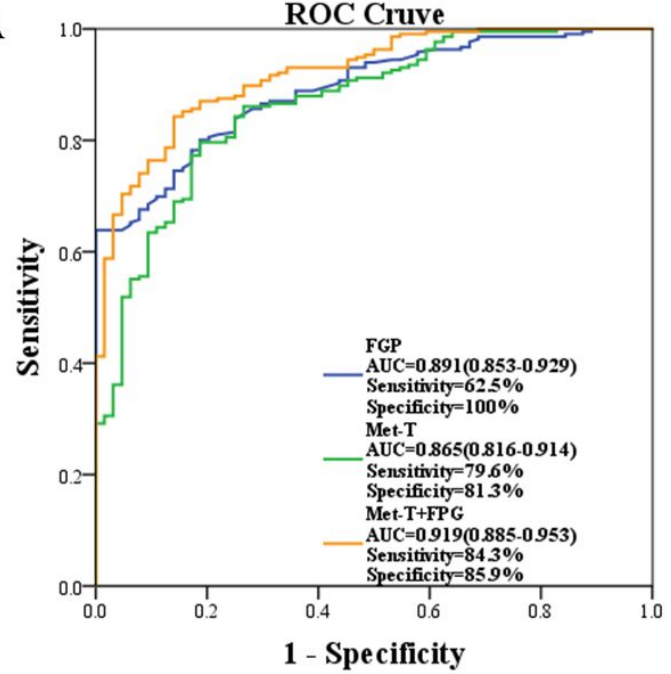

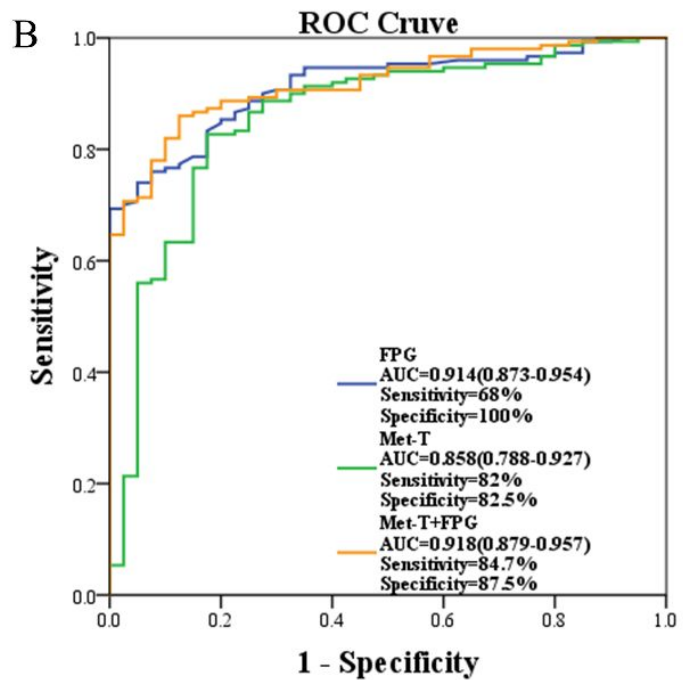

Figure S2--ROC curves of FPG, Met-T and a combination of Met-T+FPG to discriminate A and B) normal glucose tolerant individuals from prediabetes and diabetes in discovery set 2 and validation set 1 , respectively. 

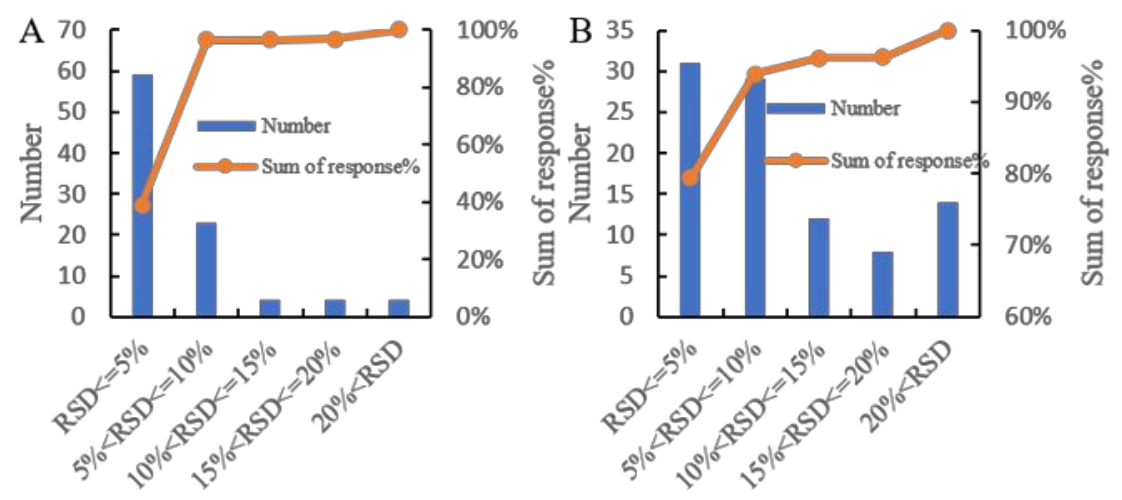

Figure S3--Analytical accuracy of the LC-MS/MS method developed for the profiling of 108 diabetes related metabolites. Shown are the distributions of the relative standard deviations (RSD). A) Intra-day precision, B) Inter-day precision. 
Table S1--Details of the references used to extract 108 (pre)diabetes related metabolites for knowledge-based metabolite profiling. The first 8 references are own previous publications.

\begin{tabular}{|c|c|c|c|c|c|}
\hline Reference & Study population & $\begin{array}{l}\text { Sample } \\
\text { material }\end{array}$ & $\begin{array}{l}\text { Analytical } \\
\text { method }\end{array}$ & Disease & $\begin{array}{c}\text { (Pre)diabetes } \\
\text { related metabolites }\end{array}$ \\
\hline $\begin{array}{l}\text { Chang Wang, et al. Anal. Chem. } \\
\text { 2005;77:4108-4116 }\end{array}$ & $69(35$ control/34 T2D $)$ & plasma & LC-MS & $\mathrm{T} 2 \mathrm{D}$ & PE16:0/22:6, PE18:0/20:4, LPC16:0, LPC18:0 \\
\hline $\begin{array}{c}\text { Kailong Yuan, et al. J. } \\
\text { Chromatogr. B 2007;850:236- } \\
240\end{array}$ & $54(26$ control/28 T2D) & urine & GC-MS & T2D & $\begin{array}{c}\text { maleic acid dimethyl ester, oxyl acetic acid, 4-aminobenzoic } \\
\text { acid, 2,5-bisoxy-benzeneacetic acid }\end{array}$ \\
\hline $\begin{array}{l}\text { Xiang Li, et al. Anal. Chim. Acta } \\
\text { 2009;633:257-262 }\end{array}$ & $\begin{array}{c}\text { 79(31 control/48 } \\
\text { diabetes })\end{array}$ & plasma & $\mathrm{GC} \times \mathrm{GC}-\mathrm{MS}$ & diabetes & $\begin{array}{c}\text { ( } \uparrow \text { glucose, C6 sugar, palmitic acid, phosphate, } \\
\text { 2-hydroxyisobutyric acid, linoleic acid }\end{array}$ \\
\hline $\begin{array}{l}\text { Xinjie Zhao,et al. Metabolomics } \\
\text { 2010;6:362-374 }\end{array}$ & $51(39$ control/12 IGT) & $\begin{array}{c}\text { plasma/urin } \\
\text { e }\end{array}$ & LC-MS & IGT & $\begin{array}{l}\text { ( } \uparrow \text { ) glycochenadeoxycholic acid, fatty acids, xanthine, } \\
\text { tryptophan, C8:2-OH carnitine, C10:2-OH carnitine ( } \downarrow \text { ) } \\
\text { LPCs, uric acid, 7-methylxanthine, methyluric acid, } \\
\text { 3-methylxanthine, 1-methylxanthine, 3-hydroxyhippuric acid, }\end{array}$ \\
\hline
\end{tabular}


(个)SM16:0, LPC18:0, SM16:1, adenosine monophosphate,

Qiang Huang, et al. J.

Chromatogr. B 2011;879:961-

967

LPE20:0, PE36:2, LPE22:0, PC33:2, PC31:0, PC40:6,

LPC15:0, PC38:7, PC36:1, PC36:2, PC38:6, glycocholic acid, FFA18:0 ( $\downarrow$ ) PC32:1, palmitoylcarnitine, LPE22:5, vaccenylcarnitine, LPC16:1, LPC22:5, LPE18:2, LPE20:4,

PC38:5, LPC18:2, FFA22:5, FFA22:4, ascorbic acid, before exercise: $(\uparrow)$ lactate, glutamate, LPC 14:0, LPC16:1,

Jakob S. Hansen, et al.

Diabetologia 2015;58:1845-1854

Qiuhui Xuan, et al. Anal. Chem.

2018;90:7608-7616

$60(30$ control $/ 30$

diabetes)

serum

LC-MS

diabetes

Lichao Wang, et al. Anal. Chem.

2018;90:11401-11408

15(8 control/7 T2D) plasma LC-MS T2D
LPC18:3, LPC20:3, FFA 14:0, 15:0, 16:0, 18:0, acylcarnitine, carnitine 14:0 $(\downarrow)$ arginine,

asparagine, cysteine, glutamine, glycine, threonine, LPC 18:0

( $\uparrow$ TAGs, DAGs, PEs, LPEs $(\downarrow)$ HexCers, PE-Os, PC-Os,

LPC-Os

$(\uparrow)$ acetylcarnitine/carnitine $(\downarrow) 2$-seoxy-galactose, carnitine 10:0, carnitine 10:1 
( $\uparrow$ acyl:free ratio, acetylcarnitine, hexanoylcarnitine,

Sean H. Adams, et al. J. Nutr

2009;139:1073-108

$56(12$ control $/ 44$

diabetes)
LC-MS plasma

T2D

octanoylcarnitine, decanoylcarnitine,

3,4-methylene-nonanoylcarnitine, myristoylcarnitine,

\begin{tabular}{|c|c|c|c|c|c|}
\hline & & & & & $\begin{array}{l}(\uparrow) \text { trimethylamine, dimethylamine, dimethylglycine, betaine, } \\
\text { trimethylamine-N-oxide, citrate, butyrate, hydroxybutyrate, }\end{array}$ \\
\hline $\begin{array}{l}\text { R.M. Salek, et al. Physiol } \\
\text { Genomics 2007;29:99-108 }\end{array}$ & $\begin{array}{l}42(12 \text { control } / 30 \mathrm{~T} 2 \mathrm{D}) \\
\& 16 \text { rats }(8 \text { control } / 8 \\
\mathrm{T} 2 \mathrm{D})\end{array}$ & urine & NMR/LC-MS & T2D & $\begin{array}{c}\text { glutamine, taurine, N-methylnicotinamide } \\
\text { N-methyl-2-pyridone-5-carboxamide }(\downarrow) \text { creatine, creatinine, } \\
\text { 2-oxoglutarate, } \mathrm{N} \text {-methylnicotinate, allantoin; malate, } \\
\text { fumarate, succinate }(\mathrm{r} \uparrow, \mathrm{h} \downarrow) \text {; acetate, acetoacetate, alanine, } \\
\text { ornithine }(\mathrm{r} \downarrow, \mathrm{h} \uparrow) \text {; }\end{array}$ \\
\hline $\begin{array}{l}\text { E. S. Tai, et al. Diabetologia } \\
\qquad 2010 ; 53: 757-767\end{array}$ & $\begin{array}{l}\text { 180(97 low HOMA/83 } \\
\text { high HOMA) \& 83(30 } \\
\text { low HOMA/53 high } \\
\text { HOMA) }\end{array}$ & $\begin{array}{l}\text { plasma/ } \\
\text { urine }\end{array}$ & $\begin{array}{l}\text { LC-MS/GC- } \\
\qquad \text { MS }\end{array}$ & IR & $\begin{array}{l}(\uparrow) \text { alanine, proline, valine, leucine/isoleucine, phenylalanine, } \\
\text { tyrosine, glutamate/glutamine, ornithine, lactate, pyruvate }\end{array}$ \\
\hline $\begin{array}{l}\text { Xiuying Zhang,er al. J. Proteome } \\
\text { Res. 2009;8:5188-5195 }\end{array}$ & $\begin{array}{l}231(80 \text { control/77 } \\
\text { IFG/74 T2D) }\end{array}$ & serum & NMR & IFG/T2D & $\begin{array}{l}(\uparrow) \text { glucose }(\downarrow) \text { isoleucine, leucine, valine, alanine, } \\
\text { methionine, glutamine, citrate, lysine, choline, lactate, } \\
\text { tyrosine, phenylalanine, histidine }\end{array}$ \\
\hline
\end{tabular}


( $\uparrow$ mucic acid, phosphocreatine, creatinine, glycine, guanidinoacetate, malonate, carnitine, $\mathrm{N}$-acetylglutamate, choline, threonine, glucose, lactate, alanine, acetate, formate, 2-oxoadipipate, lysine, methionine, N1-methylnictinamide,

N1-methylnicotinic acid, nicotinamide-n-oxide,

N-methyl-2-pyridone-5-carboxamide,

N-methyl-4-pyridone-5-carboxamide, pantothenate,

3-ureidopropanoate, allantion, orotate, sucrose, malate,

Susan C. Connor, et al. Mol.

BioSyst. 2010;6:909-921
20 mice(10 control/10

diabetes) urine

NMR

diabetes

citrulline, L-argininosuccinic acid, $\mathrm{N}$-acetyl citrulline,

ornithine, 2-hydroxyisobutyrate, 2-hydroxyisovalerate, valine

$(\downarrow) \mathrm{N}$-acetylasparate, butyrylglycine, 2-hydroxy-N-valerate, caproylglycine, valeryglycine, glutaric acid, isocaproylglycine, cis-aconitate, taurine, indoxyl sulfate,

2-oxo-3-methyl-N-valerate, 2-oxoisocaproate,

2-oxoisovalerate, isobutyrlglycine, isovalerate, isovalerylglycine, methylmalonate 


\begin{tabular}{|c|c|c|c|c|c|}
\hline $\begin{array}{l}\text { Walter E. Gall, et al. Plos one } \\
\qquad 2010 ; 5: \mathrm{e} 10883\end{array}$ & $\begin{array}{l}\text { 399(211 NGT-IS/45 } \\
\text { NGT-IR/82 IGT/61 } \\
\text { IFG) }\end{array}$ & plasma & $\begin{array}{l}\text { LC-MS/GC- } \\
\qquad \mathrm{MS}\end{array}$ & IR & $\begin{array}{c}(\uparrow) \text { alpha-hydroxybutyrate, 3-methyl-2-oxobutyrate, creatine, } \\
\text { adrenate, stearate, palmitate, margarate }(\downarrow) \\
\text { linoleoylglycerophosphocholine, glycine, } \\
\text { oleoylglycerophosphocholine, decanoylcarnitine, } \\
\text { octanylcarnitine, stearoylglycerophosphocholine, } \\
\text { palmitoylglycerophosphocholine }\end{array}$ \\
\hline $\begin{array}{l}\text { Karsten Suhre, et al. Plos one } \\
\qquad 2010 ; 5: \mathrm{e} 13953\end{array}$ & $\begin{array}{l}100(60 \text { control } / 40 \\
\text { T2D) }\end{array}$ & $\begin{array}{l}\text { serum/plas } \\
\text { ma }\end{array}$ & $\begin{array}{l}\text { NMR/LC-MS } \\
\text { /GC-MS }\end{array}$ & diabetes & $\begin{array}{l}\text { ( } \uparrow \text { desoxyhexose, glucose, H3-HNAc2-NANA, HNAC, } \\
\text { HNAc-H2-dH, uronic acid, dihexose, mannose, creatinine, } \\
\text { glutamylvaline, glutamylisoleucine, 3-hydroxxybutyrate, } \\
\text { phenylacetylglutamine, phenylalanine, 3-indoxyl sulfate, } \\
\text { kynurinine, homocitrulline, isoleucine, leucine, } \\
\text { N-acetylglucosamine, dexxyglucose, glucuronic acid ( } \downarrow \text { ) } \\
\text { 1,5-anhydroglucitol, caproate, heptanoate, pelargonate, } \\
\text { glycerophosphorylcholine, PC a 20:4, PC aa (OH,COOH) } \\
\text { 28:4, PC aa 34:4, SM 14:0, SM 22:2 }\end{array}$ \\
\hline $\begin{array}{l}\text { Andrew D. Patterson, et al. J. } \\
\text { Biol. Chem. 2011;286:19511- } \\
\qquad 19522\end{array}$ & $\begin{array}{l}21 \text { monekey(10 } \\
\text { control/11 T2D) }\end{array}$ & urine & LC-MS & $\mathrm{T} 2 \mathrm{D}$ & $\begin{array}{l}\text { ( } \uparrow \text { glycine betaine, citric acid, glucose, kynurenic acid, } \\
\text { pipecolic acid, proline }\end{array}$ \\
\hline
\end{tabular}




\begin{tabular}{|c|c|c|c|c|c|}
\hline $\begin{array}{l}\text { Thomas J. Wang, et al. Nat. Med. } \\
\text { 2011;17:448-454 }\end{array}$ & $\begin{array}{l}\text { 778(189 control/189 } \\
\text { diabetes/400 random } \\
\text { control })\end{array}$ & plasma & LC-MS & diabetes & $(\uparrow)$ isoleucine, leucine, valine, tyrosine, phenylalanine \\
\hline $\begin{array}{l}\text { Chao Zhu, et al. Talanta } \\
\text { 2011;85:1711- } 1720\end{array}$ & $\begin{array}{l}112(30 \text { control/30 } \\
\text { T2D/52 diabetic } \\
\text { nephropathy) }\end{array}$ & plasma & LC-MS & $\begin{array}{l}\text { T2D/diabet } \\
\text { ic } \\
\text { nephropath } \\
\text { y }\end{array}$ & 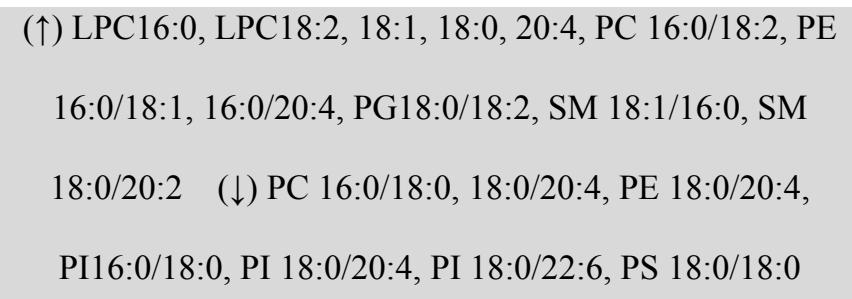 \\
\hline $\begin{array}{l}\text { Susan Cheng, et al. Circulation } \\
\text { 2012;125:2222-2231 }\end{array}$ & $\begin{array}{l}1015(414 \text { control/601 } \\
\text { diabetes) \& 746(337 } \\
\text { control/409 diabetes) }\end{array}$ & plasma & LC-MS & IR/diabetes & $(\uparrow)$ glutamate $(\downarrow)$ glutamine, glutamine-glutamate ratio \\
\hline $\begin{array}{l}\text { Changyoung Ha, et al. Clin. } \\
\text { Endocrinol. 2012;76:674-682 }\end{array}$ & $53(27$ control/26 T2D) & plasma & LC-MS & $\mathrm{T} 2 \mathrm{D}$ & $\begin{array}{l}\text { ( } \uparrow \text { ) leucine, lysine, phenylalanine, propionylcarnitine, } \\
\text { octanoylcarnitine, decanoylcarnitine, dodecanoylcarnitine, } \\
\text { palmitylcarnitine, heptadecanoylcarnitine, linoleylcarnitine, } \\
\text { vaccenylcarnitine, LPC14:0, LPC16:1, LPC18:1, LPC18:3, } \\
\text { LPC20:5, LPC22:6, LPE18:2, LPE22:6 ( } \downarrow \text { ) serine, LPE18:1 }\end{array}$ \\
\hline
\end{tabular}

Peter Würtz, et al. Diabetes

2012;61:1372-1380
7098

serum
NMR

IR
( $\uparrow$ ) leucine, isoleucine, valine, phenylalnine, tyrosine, alanine,

phosphoglycerides $(\downarrow)$ glutamine, acetoacetate, 


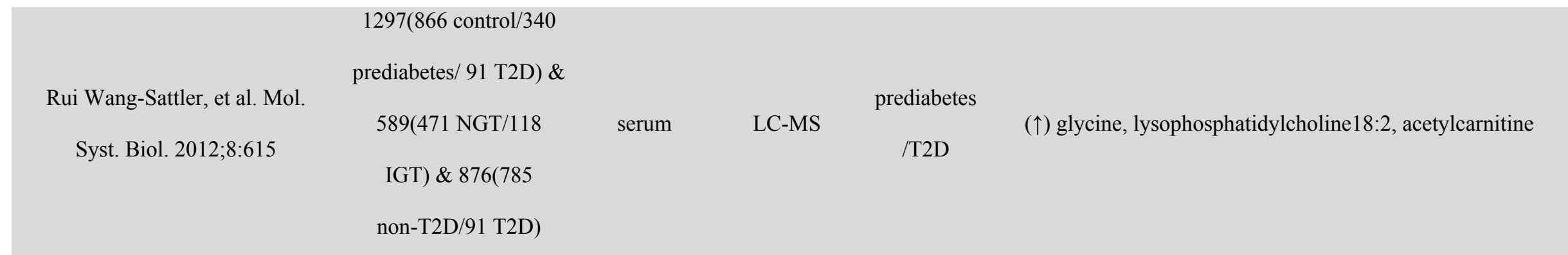

( $\uparrow$ hydroxyproline, glutamine, ethanolamine, citrulline, sarcosine, $\beta$-alanine, glutamic acid, 3-methyl histidine, $\gamma$ aminobutyric acid, $\beta$-aminoisobutyric acid, proline $(\downarrow)$

Yong Zhou, et al. Clin. Biochem. 2013;46:1447-1452 $226(100 \mathrm{control} / 126$ diabetes) plasma LC-MS diabetes phosphoserine, phosphoric acid ethanolamine, taurine, serine, aspartic acid, histidine, 1-methylhistidine, arginosuccinic acid, carnosine, anserine, $\alpha$-aminoadipic acid,

$\delta$-hydroxylysine, lysine, homocysteine, leucine, tryptophan
Anna Floegel, et al. Diabetes

$2013 ; 62: 639-648$
3082(2282 control/800

T2D)
( $\uparrow$ hexose, phenylalanien, diacyl-phosphatidylcholine C32:1, C36:1, C38:3, C40:5 ( $\downarrow)$ glycine, sphingomyelin C16:1, acyl-alkyl-phosphatidylcholine C34:3, C40:6, C42:5, C44:4, 


\section{$1308(1115$ control/146}

\section{IGR/47 T2D) \&}

Ele Ferrannini, et al. Diabetes

$2013 ; 62: 1730-1737$ plasma

LC-MS

IGR) \& $902(779$

control/123 T2D)

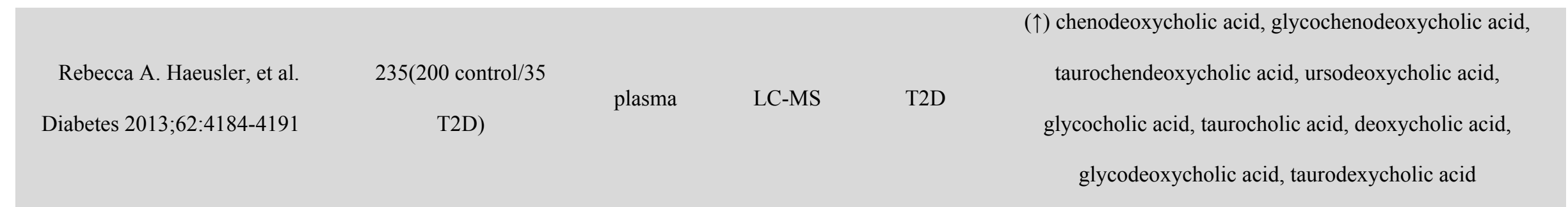

( $\uparrow$ 2-hydroxybutyrate, proline, isoleucine, leucine, valine, lactate, malate, urate, 3-methyl-2-oxobutyrate,

Cristina Menni, et al. Diabetes

$2013 ; 62: 4270-4276$

\section{4(1897 control/192}

IFG/115 T2D) plasma

\section{IGR/T2D}

linoleoylglycerophosphocholine

hyl-2-oxovalerate, 4-methyl-2-oxopentanoate, fructose,

mannose, glucose, arabinose, adrenate, arachidonate,

erythritol $(\downarrow) \mathrm{N}$-acetylglycine, citrulline,

dimethylargine,1,5-anhydroglucitol, octanoylcarnitine, 
15-methylpalmitate, 10-hepatadecenoate, myristate, myristoleate, palmitoleate, pentadecanoate, 5-dodecenoate, heptanoate, pelargonate, palmitoyl sphigomyelin, cholesteril

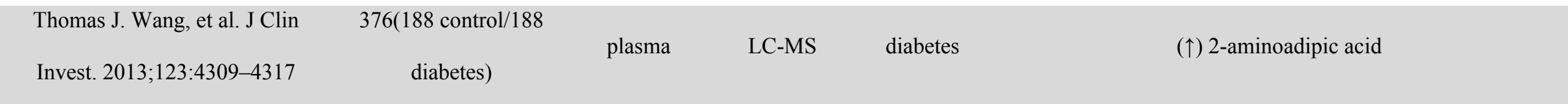

( $\uparrow$ itaconic acid, leucine, PC18:0, sphingosine-1-phosphate,

PG (18:0/18:1), N-acetyl-phenylalnine, serotonin $(\downarrow)$ inosine,

Prabhjit Kaur, et al. Mol.

BioSyst. 2013;9:307-317
182(77 control/105

$\mathrm{T} 2 \mathrm{D})$ plasma/urin

e
LC-MS

T2D

uric acid, 3-hydroxymethylglutaric acid, succinate, taurine,

PE16:0/22:6, 2-ketobutyric acid, 2-ketoglutaric acid,

1-methylhistidine, kynurenic acid, xanthurenic acid, pyruvic

\begin{tabular}{|c|c|c|c|c|c|}
\hline Anna E. Thalacker-Mercer, et al. & $124(61$ control $/ 32$ & serum & FIA-MS & $\mathrm{IR} / \mathrm{T} 2 \mathrm{D}$ & $(\uparrow)$ leucine/isoleucine, valine, aspartate/asparagines, \\
\hline Diabetes 2014;63:791-800 & $\mathrm{IR} / 31 \mathrm{~T} 2 \mathrm{D})$ & & & & glutamate/glutamine $\quad(\downarrow)$ glycine, serine, histidine \\
\hline Geoffrey A. Walford, et al. & $1622(1416 \mathrm{control} / 206$ & & & & $(\uparrow)$ tyrosine, phenylalanine, TAG48:0, TAG52:1, TAG56:9 \\
\hline Diabetes Care 2014;37:2508- & $\mathrm{T} 2 \mathrm{D})$ & plasma & LC-MS & $\mathrm{T} 2 \mathrm{D}$ & $(\downarrow)$ isoleucine, LPC18:2, PC38:6, TAG44:1 \\
\hline
\end{tabular}




\begin{tabular}{|c|c|c|c|c|c|}
\hline $\begin{array}{l}\text { Ai-hua Zhang, et al. J. Physiol. } \\
\text { Biochem. 2014;70:117-128 }\end{array}$ & $\begin{array}{l}123(42 \text { control } / 81 \\
\text { T2D) }\end{array}$ & urine & LC-MS & $\mathrm{T} 2 \mathrm{D}$ & $\begin{array}{l}\text { ( } \uparrow \text { ) adiponectin, acylcarnitines, 3-indoxyl sulfate, bile acids, } \\
\text { glucose, glycine }(\downarrow) \text { citric acid, kynurenic acid, urate, } \\
\text { glucuronolactone, lysine, phosphate }\end{array}$ \\
\hline $\begin{array}{l}\text { Martin Magnusson, et al. } \\
\text { Diabetes 2015;64:3010-3016 }\end{array}$ & 709 & plasma & LC-MS & $\begin{array}{l}\text { high } \\
\text { glucose } \\
\text { level }\end{array}$ & $(\downarrow)$ dimethylglycine, glycine \\
\hline $\begin{array}{l}\text { Jiao Sun, et al. Int. J. Diabetes } \\
\text { Dev. Ctries. 2015;35:368-373 }\end{array}$ & $\begin{array}{c}93(65 \text { control } / 28 \\
\text { diabetes })\end{array}$ & serum & ELISA & IGT/T2D & ( $\uparrow$ ) 8-hydro-2'-deoxyguanosine \\
\hline $\begin{array}{l}\text { Norihide Yokoi, et al. } \\
\text { Metabolomics 2015;11:1277- } \\
1286\end{array}$ & rat model of T2D & serum & GC-MS & prediabetes & $\begin{array}{c}(\uparrow) \text { glycerol, taurine }(\downarrow) \text { asparagine, glutamine, kynurenine, } \\
\text { mannose, n-alpha-acetyllysine, threonine, tryptophan }\end{array}$ \\
\hline $\begin{array}{l}\text { Tonia C. Carter, et al. } \\
\text { Metabolism 2016;65:1399-1408 }\end{array}$ & $\begin{array}{l}139(78 \text { control/ } 61 \\
\text { T2D) \& 501(445 } \\
\text { control/56 T2D) }\end{array}$ & plasma & $\begin{array}{l}\text { LC-MS/GC- } \\
\qquad \text { MS }\end{array}$ & $\mathrm{T} 2 \mathrm{D}$ & $\begin{array}{l}\text { ( } \uparrow \text { glucose, mannose, glucosamine, 2-hydroxybutyrate, } \\
\text { glyoxylate, glycochenodeoxycholic acid, } \\
\text { taurochenodeoxycholic acid, } \quad(\downarrow) \text { cholesteryl ester (18:1), } \\
\text { lysophosphatidylcholine (18:2), lignoceric acid (24:0) }\end{array}$ \\
\hline Gopal Peddinti, et al. & $543(397$ control $/ 146$ & serum & LC-MS/GC- & $\mathrm{T} 2 \mathrm{D}$ & ( $\uparrow$ glucose, mannose, $\alpha$-hydroxybutyric acid, isoleucine, \\
\hline
\end{tabular}


valine, glutamate, trehalose $(\downarrow)$ histidine, billrubin, glutamine,

$\alpha$-tocopherol

\begin{tabular}{|c|c|c|c|c|c|}
\hline $\begin{array}{l}\text { Tali Ganz, et al. Diabetes Metab } \\
\text { Res Rev. 2017;33:e2836 }\end{array}$ & $\begin{array}{l}242(137 \text { control } / 105 \\
\text { T2D) }\end{array}$ & serum & & $\mathrm{T} 2 \mathrm{D}$ & $(\uparrow)$ arginine \\
\hline $\begin{array}{l}\text { Otto Savolainen, et al. Plos one } \\
\qquad 2017 ; 12: \mathrm{e} 0177738\end{array}$ & $\begin{array}{l}662(188 \text { control/203 } \\
\text { IGT/202 T2D/69 new } \\
\text { T2D) }\end{array}$ & plasma & GC-MS & T2D & $\begin{array}{l}(\uparrow) \text { sorbitol, galacticol, mannose, galactose, uric acid, oxalic } \\
\text { acid, glucaric acid-1,4-lactone, 3-methyl-2-oxopentanoic } \\
\text { acid, 2-hydroxybutyric acid }\end{array}$ \\
\hline $\begin{array}{l}\text { Siming Wang, et al. Clin. Chim. } \\
\text { Acta } 2018 ; 487: 60-65\end{array}$ & $\begin{array}{l}563 \text { (429 control/134 } \\
\text { IFG/diabetes) }\end{array}$ & serum & LC-MS & $\begin{array}{l}\text { IFG/diabet } \\
\text { es }\end{array}$ & $\begin{array}{l}(\uparrow) \text { glutamic acid, valine, leucine, isoleucine, phenylalanine } \\
(\downarrow) \text { glutamine/glutamic acid, LPC18:1, LPC18:2 }\end{array}$ \\
\hline $\begin{array}{l}\text { Jordi Merino, et al. Diabetologia } \\
\qquad 2018 ; 61: 1315-1324\end{array}$ & $\begin{array}{l}1150(1055 \text { control } / 95 \\
\text { T2D) }\end{array}$ & plasma & LC-MS & $\mathrm{T} 2 \mathrm{D}$ & $\begin{array}{l}\text { SM24:0, DAG36:1, TAG58:11, 5-hydroxyindoleacetic acid, } \\
\text { PC36:4, 3-methyladipic acid, D-glucose, 2-aminoadipate, } \\
\text { isocitrate, L-phenylalanine, LPC18:2, glycine, TAG52:1, } \\
\text { LPC18:1, TAG48:1, TAG48:0, CE20:3, Taurine, TAG54:8 }\end{array}$ \\
\hline $\begin{array}{l}\text { Yonghai Lu, et al. J. Clin. } \\
\text { Endocrinol. Metab. } \\
\text { 2018;103:671-680 }\end{array}$ & $\begin{array}{c}608(304 \text { control } / 160 \\
\text { incident case } / 144 \\
\text { prevalent case) }\end{array}$ & serum & $\begin{array}{l}\text { LC-MS/GC- } \\
\qquad \mathrm{MS}\end{array}$ & $\mathrm{T} 2 \mathrm{D}$ & $\begin{array}{l}\text { (个) LPI16:0, LPI16:1, LPI18:0, LPI18:2, LPI20:4, LPI22:6, } \\
\text { ffatty acids, 3-hydroxybutyrlcarnitine, ocatanoylcarnitine, } \\
\text { decanoylcarnitine, dodecanoylcarnitine, } \\
\text { 3-hydroxydodecanoylcarnitine, tetradecanoylcarnitine, } \\
\text { 3-hydroxytetradecanoylcarnitine, hexadecanoylcarnitine, }\end{array}$ \\
\hline
\end{tabular}


3-hydroxy-9-hexadecenoylcarnitine, C20:5 carnitine ( $\downarrow$ )

myo-inositol, L-carnitine,

( $\uparrow$ ) leucine, isoleucine, valine, phenylalanine, tyrosine,

Ari V. Ahola-Olli, et al.

Diabetologia 2019;62:2298-2309
$11896(11504$

control/392 T2D) serum

$\mathrm{T} 2 \mathrm{D}$

histidine, alanine, lactate, pyruvate, glycerol, acetoacetate,

3-hydroybutyrate, docosahexaenoic acid $\quad(\downarrow)$ glycine,

glutamine, creatinine, acetate, citrate, linoleic acid,

arachidonic acid

T2D: type 2 diabetes; IR: insulin resistance; IFG: impaired fasting glucose; IGT: impaired glucose tolerance; LPC: lysophosphatidylcholine;

LPE: lysophosphatidylethanolamine PE: phosphatidylethanolamine; PC: phosphatidylcholine; PG: phosphatidylglycerole; PS:

phosphatidylserine; PI: phosphatidylinositol; SM: sphingomyelin; CE: cholesteryl ester; DAG: diacylglycerol; TAG: triacylglycerol, HexCer:

hexaglycosylceramides; LPC-O: lysophosphatidylcholine with alkyl substituents; PC-O: alkyl and alkenyl substituent phosphatidylcholine;

PE-O: alkyl and alkenyl substituent phosphatidylethanolamine. 
Table S2--List and mass spectrometric details of 108 knowledge-based selected metabolites investigated by the targeted LC-MS method in positive and negative ionization modes.

\begin{tabular}{|c|c|c|c|c|c|}
\hline Q1 & Q3 & $\operatorname{tr} / \min$ & metabolites & DP & $\mathrm{CE}$ \\
\hline 163.06 & 100.80 & 2.56 & 1,5-Anhydroglucitol & -100 & -15 \\
\hline 87.05 & 86.90 & 1.02 & Butyric acid & -60 & -15 \\
\hline 103.04 & 56.90 & 2.23 & $\begin{array}{l}\text { 2-hydroxybutyric/2-Hydroxyisobutyric } \\
\text { acid }\end{array}$ & -60 & -30 \\
\hline 117.06 & 71.00 & 1.64 & 2-Hydroxyisovaleric acid & -100 & -15 \\
\hline 188.07 & 144.08 & 0.93 & 3-Indolepropionic acid & -100 & -15 \\
\hline 212.00 & 79.90 & 0.99 & 3-Indoxyl sulfate & -100 & -45 \\
\hline 136.04 & 91.90 & 1.46 & 4-Aminobenzoic acid & -60 & -15 \\
\hline 219.08 & 144.00 & 3.37 & 5-Hydroxy-tryptophan & -80 & -30 \\
\hline 266.09 & 134.20 & 1.65 & Adenosine & -100 & -30 \\
\hline 145.05 & 81.00 & 3.96 & Adipic acid & -60 & -45 \\
\hline 145.01 & 100.90 & 4.06 & $\alpha$-Ketoglutaric acid & -60 & -15 \\
\hline 88.04 & 88.04 & 3.68 & Alanine & -80 & -10 \\
\hline 157.04 & 96.90 & 2.44 & Allantoin & -60 & -30 \\
\hline 346.06 & 78.80 & 4.40 & Adenosine monophosphate & -60 & -45 \\
\hline 149.05 & 58.80 & 2.70 & Arabinose & -60 & -30 \\
\hline 131.05 & 131.05 & 3.88 & Asparagine & -100 & -10 \\
\hline 132.03 & 88.20 & 4.20 & Aspartic acid & -60 & -30 \\
\hline 407.28 & 343.30 & 1.50 & Cholate & -60 & -45 \\
\hline 191.02 & 191.02 & 5.43 & Citric acid & -100 & -30 \\
\hline 174.09 & 131.00 & 3.98 & Citrulline & -60 & -30 \\
\hline 130.06 & 88.00 & 3.55 & Creatine & -60 & -15 \\
\hline 112.05 & 68.00 & 1.73 & Creatinine & -100 & -15 \\
\hline 259.02 & 78.80 & 4.46 & Fructose-6-phosphate & -60 & -45 \\
\hline 102.06 & 101.90 & 2.98 & Dimethylglycine & -60 & -10 \\
\hline
\end{tabular}




\begin{tabular}{|c|c|c|c|c|}
\hline 115.00 & 70.90 & 4.19 & Fumaric acid & -60 \\
\hline 209.03 & 85.00 & 5.42 & Galactaric acid & -100 \\
\hline 179.06 & 59.00 & 3.44 & Hexose & -80 \\
\hline 193.04 & 58.90 & 4.08 & Glucuronic acid & -60 \\
\hline 72.99 & 72.99 & 3.45 & Glyoxylate & -60 \\
\hline 116.05 & 73.90 & 3.70 & Guanidinoacetic acid & -60 \\
\hline 118.05 & 54.90 & 3.82 & Homoserine & -60 \\
\hline 174.06 & 130.07 & 1.74 & Indole-3-acetic acid & -100 \\
\hline 179.06 & 87.00 & 3.83 & Inositol & -100 \\
\hline 89.02 & 89.02 & 2.86 & Lactic acid & -60 \\
\hline 104.04 & 74.00 & 4.06 & Serine & -60 \\
\hline 133.01 & 71.20 & 4.00 & Malate & -100 \\
\hline 178.07 & 58.80 & 4.11 & Mannosamine & -60 \\
\hline 174.04 & 88.00 & 4.25 & N-Acetyl-aspartic acid & -60 \\
\hline 218.10 & 88.00 & 2.85 & Pantothenic acid & -100 \\
\hline 87.01 & 87.01 & 2.26 & Pyruvic acid & -60 \\
\hline 149.05 & 59.10 & 2.13 & Ribose & -60 \\
\hline 173.08 & 111.00 & 3.58 & Suberic acid & -80 \\
\hline 117.02 & 73.10 & 3.97 & Succinic acid & -60 \\
\hline 118.05 & 74.10 & 3.79 & Threonine & -60 \\
\hline 180.07 & 93.10 & 3.26 & Tyrosine & -80 \\
\hline 167.02 & 69.10 & 3.33 & Uric acid & -80 \\
\hline 151.03 & 108.00 & 1.88 & Xanthine & -60 \\
\hline 411.31 & 347.30 & 1.42 & Cholic acid-d4 & -60 \\
\hline 150.09 & 131.10 & 3.83 & Glutamine-d5 & -60 \\
\hline 183.08 & 82.00 & 2.00 & Hippuric acid-d5 & -60 \\
\hline 169.10 & 152.10 & 2.53 & Phenylalanine-d5 & -100 \\
\hline 121.10 & 121.10 & 3.19 & Proline-d7 & -100 \\
\hline 121.04 & 76.10 & 4.07 & Succinic acid-13C4 & -60 \\
\hline 162.08 & 98.00 & 4.31 & 2-Aminoadipic acid & 80 \\
\hline
\end{tabular}




\begin{tabular}{|c|c|c|c|c|}
\hline 118.09 & 101.00 & 4.17 & 5-Amino pentanoic acid & 100 \\
\hline 284.10 & 168.00 & 2.52 & 8-Hydroxy-2 -deoxyguanosine & 100 \\
\hline 118.09 & 58.10 & 2.70 & Betaine & 100 \\
\hline 162.11 & 85.02 & 3.54 & Carnitine & 100 \\
\hline 316.25 & 85.02 & 1.30 & Decanoylcarnitine & 100 \\
\hline 314.23 & 85.02 & 1.50 & Decenoylcarnitine & 100 \\
\hline 310.20 & 85.02 & 1.80 & Decatrienoylcarnitine & 100 \\
\hline 344.28 & 85.02 & 1.36 & Dodecanoylcarnitine & 100 \\
\hline 372.31 & 85.02 & 1.29 & Myristoylcarnitine & 100 \\
\hline 370.30 & 85.02 & 1.30 & Tetradecenoylcarnitine & 100 \\
\hline 400.34 & 85.02 & 1.21 & Palmitoylcarnitine & 100 \\
\hline 414.36 & 85.02 & 1.17 & Heptadecanoylcarnitine & 100 \\
\hline 428.37 & 85.02 & 1.13 & Octadecanoylcarnitine & 100 \\
\hline 426.36 & 85.02 & 1.20 & Vaccenylcarnitine & 100 \\
\hline 424.34 & 85.02 & 1.25 & Linoleylcarnitine & 100 \\
\hline 204.12 & 85.02 & 2.85 & Acetylcarnitine & 100 \\
\hline 218.14 & 85.02 & 2.48 & Propionylcarnitine & 100 \\
\hline 232.15 & 85.02 & 2.18 & Butyrylcarnitine & 100 \\
\hline 230.14 & 85.02 & 2.20 & Butenoylcarnitine & 100 \\
\hline 246.17 & 85.02 & 1.98 & Isovalerylcarnitine & 100 \\
\hline 260.19 & 85.02 & 1.86 & Hexanoylcarnitine & 100 \\
\hline 288.22 & 85.02 & 1.57 & Octanoylcarnitine & 100 \\
\hline 286.20 & 85.02 & 1.60 & 2-Octenoylcarnitine & 100 \\
\hline 104.11 & 60.10 & 2.80 & Choline & 100 \\
\hline 363.22 & 121.10 & 0.59 & Cortisol & 60 \\
\hline 62.06 & 44.00 & 3.80 & Ethanolamine & 60 \\
\hline 180.09 & 162.20 & 4.22 & Glucosamine + mannosamine & 80 \\
\hline 148.06 & 55.60 & 4.12 & Glutamic acid & 60 \\
\hline 147.08 & 84.00 & 3.86 & Glutamine & 80 \\
\hline 76.04 & 30.30 & 3.89 & Glycine & 60 \\
\hline
\end{tabular}




\begin{tabular}{|c|c|c|c|c|}
\hline 466.32 & 412.28 & 2.45 & Glycocholic acid & 100 \\
\hline 180.07 & 104.90 & 2.01 & Hippuric acid & 100 \\
\hline 156.08 & 110.20 & 6.30 & Histidine & 60 \\
\hline 120.07 & 56.10 & 3.81 & Homoserine + threonine & 60 \\
\hline 132.10 & 86.10 & 2.75 & Isoleucine & 100 \\
\hline 209.09 & 192.10 & 2.59 & Kynurenine & 100 \\
\hline 132.07 & 68.10 & 3.64 & 4-Hydroxyproline & 60 \\
\hline 241.03 & 74.00 & 4.78 & Cystine & 60 \\
\hline 132.10 & 85.80 & 2.64 & Leucine & 60 \\
\hline 147.11 & 130.30 & 5.98 & Lysine & 60 \\
\hline 524.37 & 184.07 & 1.72 & LPC 18:0 & 100 \\
\hline 522.36 & 184.07 & 1.69 & LPC 18:1 & 100 \\
\hline 345.14 & 183.00 & 3.81 & Maltitol & 60 \\
\hline 150.06 & 61.00 & 2.87 & Methionine & 80 \\
\hline 222.10 & 137.90 & 2.85 & N-Acetylglucosamine & 60 \\
\hline 190.07 & 83.90 & 4.11 & N-Acetyl-glutamic acid & 80 \\
\hline 133.10 & 70.00 & 6.20 & Ornithine & 60 \\
\hline 166.09 & 120.00 & 2.54 & Phenylalanine & 100 \\
\hline 130.09 & 84.00 & 3.07 & Pipecolic acid & 100 \\
\hline 116.07 & 70.10 & 3.20 & Proline & 100 \\
\hline 701.56 & 184.07 & 1.20 & SM 16:1 & 100 \\
\hline 755.61 & 184.07 & 1.10 & SM 20:2 & 100 \\
\hline 482.29 & 464.30 & 1.68 & Taurochenodeoxycholic acid & 80 \\
\hline 138.06 & 92.10 & 2.79 & Trigonelline & 100 \\
\hline 76.08 & 57.90 & 3.60 & Trimethylamine-n-oxide & 100 \\
\hline 205.10 & 188.00 & 2.67 & Tryptophan & 100 \\
\hline 245.08 & 113.10 & 1.77 & Uridine & 60 \\
\hline 118.09 & 72.10 & 3.05 & Valine & 100 \\
\hline 265.12 & 84.00 & 2.45 & N-Phenylacetyl-glutamine & 100 \\
\hline 431.39 & 165.10 & 0.47 & Tocopherol & 80 \\
\hline
\end{tabular}




\begin{tabular}{cccccc}
319.27 & 85.00 & 1.30 & Decanoylcarnitine-d3 & 100 & 45 \\
207.14 & 85.00 & 2.88 & Acetylcarnitine-d3 & 60 & 45 \\
377.30 & 359.20 & 1.42 & Cholic acid-d4 & 100 & 15 \\
108.13 & 60.20 & 2.84 & Choline-d4 & 100 & 15 \\
152.11 & 88.10 & 3.83 & Glutamine-d5 & 80 & 30 \\
185.10 & 110.20 & 2.00 & Hippuric acid-d5 & 100 & 15 \\
171.12 & 125.10 & 2.53 & Phenylalanine-d5 & 100 & 30 \\
\hline
\end{tabular}


Table S3--Detailed information about IS in methanol

\begin{tabular}{cc}
\hline IS & Concentration in methanol $\mu \mathrm{g} / \mathrm{mL}$ \\
\hline cholic acid-d4 & 0.23 \\
hippuric acid-d5 & 0.19 \\
phenylalanine-d5 & 0.47 \\
glutamine-d5 & 1.56 \\
carnitine C2-d3 & 0.04 \\
carnitine C10-d3 & 0.01 \\
choline-d4 & 0.31 \\
succinic acid- ${ }^{13} \mathrm{C} 4$ & 0.63 \\
proline-d7 & 0.31 \\
\hline
\end{tabular}


Table S4--Detailed information about the method validation

\begin{tabular}{|c|c|c|c|c|c|c|c|c|}
\hline & \multirow[b]{2}{*}{ compounds } & \multicolumn{2}{|l|}{ linear } & LOD & LOQ & \multicolumn{3}{|c|}{ Recovery (\%) } \\
\hline & & $\begin{array}{c}\text { range } \\
(\mathrm{ng} / \mathrm{mL})\end{array}$ & $\mathrm{R} 2$ & \multicolumn{2}{|c|}{$(\mathrm{ng} / \mathrm{mL})(\mathrm{ng} / \mathrm{mL})$} & high & median & low \\
\hline \multirow{6}{*}{ neg } & Cholic acid-d4 & $5-10000$ & 0.9998 & 0.1 & 0.5 & $104.2 \pm 5.2$ & $97.8 \pm 6.0$ & $94.9 \pm 2.2$ \\
\hline & Hippuric acid-d5 & $5-20000$ & 0.9948 & 0.01 & 1 & $101.4 \pm 1.6$ & $101.3 \pm 3.2$ & $94.8 \pm 1.0$ \\
\hline & Phenylalanine-d5 & $5-20000$ & 0.996 & 0.1 & 0.5 & $101.1 \pm 1.3$ & $96.6 \pm 0.9$ & $94.9 \pm 1.0$ \\
\hline & Proline-d7 & $100-20000$ & 0.999 & 10 & 100 & $99.5 \pm 1.8$ & $94.9 \pm 2.2$ & $105.4 \pm 4.7$ \\
\hline & Succinic acid- ${ }^{13} \mathrm{C} 4$ & $100-20000$ & 0.9982 & 1 & 5 & $105.1 \pm 2.4$ & $92.8 \pm 4.7$ & $88.1 \pm 6.7$ \\
\hline & Glutammine-d5 & $50-20000$ & 0.9996 & 1 & 5 & $102.2 \pm 3.9$ & $96.1 \pm 1.7$ & $101.2 \pm 6.3$ \\
\hline \multirow{7}{*}{ pos } & Cholic acid-d4 & $5-20000$ & 0.9969 & 5 & 10 & $103.4 \pm 4.4$ & $98.2 \pm 7.3$ & $91.4 \pm 4.8$ \\
\hline & Acetylcarnitine-d3 & $0.005-5000$ & 0.9987 & $0.005<$ & $0.005<$ & $99.5 \pm 5.8$ & $111.8 \pm 8.1$ & $102.8 \pm 7.2$ \\
\hline & Decanoylcarnitine-d3 & $0.005-5000$ & 0.9941 & $0.005<$ & $0.005<$ & $105.7 \pm 3.0$ & $99.9 \pm 3.4$ & $99.1 \pm 2.1$ \\
\hline & Hippuric acid-d5 & $1-20000$ & 0.9992 & 0.01 & 0.1 & $102.2 \pm 3.3$ & $96.1 \pm 3.2$ & $96.0 \pm 2.0$ \\
\hline & Phenylalanine-d5 & $5-20000$ & 0.9967 & 0.1 & 0.5 & $102.4 \pm 1.8$ & $100.5 \pm 1.8$ & $96.2 \pm 2.9$ \\
\hline & Choline-d4 & $5-10000$ & 0.9948 & 0.1 & 0.5 & $102.2 \pm 0.8$ & $100.5 \pm 0.6$ & $95.6 \pm 2.6$ \\
\hline & Glutamine-d5 & $10-20000$ & 0.9997 & 2 & 5 & $103.2 \pm 1.6$ & $100.2 \pm 2.6$ & $96.2 \pm 7.1$ \\
\hline
\end{tabular}

Entries are presented as mean \pm SD. neg: negative ion mode, pos: positive ion mode. 